La revue La revue pour l'histoire du CNRS

POUR LHISTORE DU CNRS $\quad 18 \mid 2007$

Voyages collectifs en géographie

\title{
Les géographes et le travail collectif
}

La recherche coopérative sur programme à l'œuvre

Olivier Orain et Marie-Pierre Sol

\section{(2) OpenEdition}

Journals

Édition électronique

URL : https://journals.openedition.org/histoire-cnrs/4061

DOI : $10.4000 /$ histoire-cnrs.4061

ISSN : 1955-2408

Éditeur

CNRS Éditions

Édition imprimée

Date de publication : 3 octobre 2007

ISBN : 978-2-271-06559-9

ISSN : $1298-9800$

Référence électronique

Olivier Orain et Marie-Pierre Sol, « Les géographes et le travail collectif », La revue pour I'histoire du CNRS [En ligne], 18 | 2007, mis en ligne le 03 octobre 2009, consulté le 20 mai 2021. URL : http:// journals.openedition.org/histoire-cnrs/4061 ; DOI : https://doi.org/10.4000/histoire-cnrs.4061

Ce document a été généré automatiquement le 20 mai 2021.

Comité pour l'histoire du CNRS 


\title{
Les géographes et le travail collectif
}

La recherche coopérative sur programme à l'œuvre

\author{
Olivier Orain et Marie-Pierre Sol
}

1 Les années 1970 apparaissent comme une période d'épanouissement des collectifs, en géographie comme dans d'autres disciplines. Le CNRS a vraisemblablement joué un rôle important dans cette dynamique: il a fourni des opportunités, des crédits et un affichage à différents projets, notamment à travers les Recherches coopératives sur programme (RCP). Quoique créées en 1958, celles-ci n'ont connu un réel développement qu'à partir de 1969, du moins en géographie. Dans ce cadre, des projets de recherches ont été entrepris qui, en se pérennisant, ont contribué au renouvellement des centres d'intérêt et des manières de faire, principalement en géographie humaine.

Une décennie de créations

2 En géographie comme dans la plupart des sciences de l'homme d'institutionnalisation ancienne (lettres, philosophie, histoire) ${ }^{1}$, la recherche individuelle est longtemps demeurée la règle. Suivant cette logique, le CNRS a longtemps été considéré par le monde des facultés des lettres comme une institution de rattachement provisoire pour l'achèvement d'une thèse d'État. Néanmoins, dès les années 1950, se développait en géographie physique un travail de laboratoire inspiré des pratiques collectives qui avaient déjà cours dans les sciences naturelles (au sens large). Et à partir de 1968, sous l'impulsion de Claude Bataillon, le comité de géographie du CNRS a développé une politique de recrutement de chargés de recherche dont le projet devait consister à animer une équipe et non pas à terminer leur thèse. Depuis 1966, le CNRS offrait déjà à des universitaires l'opportunité de constituer des structures collectives de recherche labellisées : unité puis équipe de recherche associée (ERA).

Mais pour obtenir ce label et les financements liés, les collectifs universitaires devaient pouvoir justifier d'une certaine pérennité, d'une unité de lieu, d'une thématique forte et surtout d'une masse critique de chercheurs. Or, en géographie, très rares étaient les entreprises susceptibles d'obtenir cette qualification, sinon des noyaux localisés à Paris ou bénéficiant de l'attrait conféré par le prestige de la géographie physique. Pour les autres, existait la possibilité de créer des Recherches coopératives sur programme (RCP), qui devaient être portées par un professeur des universités, associé à des pairs ${ }^{2}$, chacun 
étant soutenu par le cortège de ses assistants. Les chercheurs relevant du CNRS étaient rattachés institutionnellement aux laboratoires propres ${ }^{3}$ ou associés (LA), ou aux ERA. Rares furent ceux qui participèrent directement aux RCP. Celles-ci illustrent une autre facette de l'essor des pratiques collectives: plus précaires (d'une durée de trois ans, elles ne pouvaient être renouvelées plus d'une fois) et moins bien dotées, elles avaient en revanche l'intérêt de pouvoir fédérer en réseau des groupes dispersés dans plusieurs universités, contrairement aux ERA...

Des RCP pluridisciplinaires ont ainsi fonctionné, auxquelles de nombreux géographes ont participé; elles ont pu être de grande ampleur, notamment quand elles impliquaient des missions lointaines. La première création ${ }^{4}$ propre à la discipline fut la RCP 147, "Villes et régions en Amérique latine », dirigée par Olivier Dollfus, qui dura de 1966 à 1969 et produisit un Cahier unique en 1970. En 1969, le biogéographe Gabriel Rougerie (Paris VII) lança la RCP 231 « Recherches sur les équilibres des paysages », en association avec des géographes de Besançon (Jean-Claude Wieber, Jean-Philippe Massonie, Daniel Mathieu), Jean-François Richard (Orstom) et avec le soutien d'une ERA toulousaine, le Centre interdisciplinaire de recherche sur les milieux naturels et l'aménagement rural (CIMA), dirigé par Georges Bertrand. Elle a publié quatre fascicules ronéotés et organisé un colloque, dont les actes ont été édités dans un numéro de 1972 de la Revue de géographie des Pyrénées et du Sud-Ouest (RGPSO).

5 Au début des années 1970, quatre autres projets ${ }^{5}$ ont vu le jour. En 1970, ce sont les RCP 256 , «Les systèmes d'organisation de l'espace », et 257 , «Les structures régionales du Midi de la France et de la Catalogne », portées respectivement par Roger Brunet (Reims) et Bernard Kayser (Toulouse). Nonobstant leurs dissemblances, le succès de ces deux projets pouvait sembler aléatoire : le groupe toulousain s'était vu auparavant (à la fin des années 1960) refuser la création d'une ERA pour le Centre interdisciplinaire d'études urbaines (CIEU), tandis que les centres d'intérêt du collectif strasbourgorémois étaient particulièrement audacieux pour l'époque. En outre, il s'agissait de projets exclusivement centrés sur la géographie humaine, laquelle n'avait pas alors un grand prestige scientifique.

6 Il a fallu le fort appui de patrons en vue (Étienne Juillard) et de membres du comité de géographie (Jacqueline Beaujeu-Garnier, C. Bataillon) pour que ces projets aboutissent. La RCP 256 a été installée avec pour horizon la fédération des recherches dans le Nord de la France ${ }^{6}$, la 257 jouant un rôle équivalent dans le Sud ; au pôle toulousain étaient associés des géographes des universités d'Aix-Marseille, de Bordeaux, de Montpellier, de Pau et de Barcelone ${ }^{7}$. Il était également tenu pour acquis qu'elles pouvaient bénéficier d'apports financiers extérieurs au CNRS et à l'Université, éventuellement importants, fournis par des travaux de "géographie appliquée». La RCP 257, reconduite jusqu'en 1976, a donné lieu à une publication aux éditions du CNRS en 1978, sous le titre Espaces périphériques.

7 En 1973, c'est la formation de la RCP 354, «Espaces vécus et civilisations », qui sera renouvelée jusqu'en 1979. Elle était pilotée par Armand Frémont (Caen), associé à Jean Gallais (Rouen) et à Michel-Jean Bertrand (Vincennes devenue Paris VIII) ${ }^{8}$. Un colloque a été organisé à Rouen à l'issue de la première période (1976), et un ouvrage collectif, Espaces vécus et civilisations, publié par les éditions du CNRS en 1982.

8 Enfin, Philippe Pinchemel a obtenu en 1976 la création de la RCP 437, «Histoire et épistémologie de la géographie ", adossée au centre de recherche qu'il avait fondé à la Sorbonne avec Michel Mollat en $1967^{9}$. Elle a assuré la responsabilité d'un numéro du 
Bulletin de la section de géographie $\left(\mathrm{n}^{\circ} 84\right)$ du Comité des travaux historiques et scientifiques, publié en 1981, et le volume 3 des Documents pour l'histoire du vocabulaire scientifique (1982), publié par l'Institut national de la langue française.

Les prémices d'un renouveau en géographie humaine

9 Il apparaît donc clairement que les Recherches coopératives sur programme ont constitué dans les années 1970 un moyen pour le CNRS de soutenir des projets centrés sur la géographie humaine, souvent originaux par rapport aux orientations de la recherche dominant jusque-là, et qui reposaient sur des effectifs ténus ou dispersés, et majoritairement provinciaux. Mais les ressources financières attribuées étaient assez maigres (entre 15000 et 30000 francs par an, dont des vacations au compte-gouttes). Une boutade de Pierre Monbeig ${ }^{10}$, rapportée par C. Bataillon, illustre la position de l'administration du CNRS: «Mes chers collègues, je voudrais que vous compreniez que [les $R C P]$ c'est pas un truc pour faire du fric mais du fric pour faire des trucs!». Au reste, les porteurs de projet se sont tous plaints de la faiblesse des moyens alloués, que ce soit dans leurs rapports ou dans les publications. Pour autant, on ne saurait dire que l'argent était le seul ressort des demandes de RCP. Il en allait en effet autant de la reconnaissance par une institution scientifique (de plus en plus) prestigieuse de projets alors situés à la périphérie de la discipline : le label conféré par le CNRS accroissait en quelque sorte la légitimité de ces entreprises.

Un regard rétrospectif est à ce titre révélateur. Les six responsables étaient de jeunes professeurs à la carrière ascendante, considérés comme des rénovateurs de la discipline. Ils ont tous laissé un nom, tout particulièrement les quatre derniers: R. Brunet a été le "capitaine d'industrie " ${ }^{11}$ de la géographie française du dernier tiers du $\mathrm{XX}^{\mathrm{e}}$ siècle, exerçant une influence durable via la revue L'Espace géographique (fondée en 1972), puis le GIP RECLUS (créé en 1983) ; B. Kayser a joué un rôle marquant dans l'engagement des géographes auprès des collectivités locales et le développement de pratiques interdisciplinaires; A. Frémont est devenu une référence après la publication de son livre La région, espace vécu (1976) ; P. Pinchemel, enfin, est, à l'instar de R. Brunet, une figure théorique majeure de la géographie française et celui qui a permis la disciplinarisation de son histoire et de son épistémologie. En outre, quatre RCP sur six augurent de la formation de courants majeurs de la géographie contemporaine, qui ont émergé durant les années 1970-1980 : géographie de l'environnement et des paysages (G. Rougerie et G. Bertrand), analyse spatiale (R. Brunet), géographie sociale (A. Frémont, J. Chevalier, certains géographes toulousains), aménagement (B. Kayser).

11 Si les publications des RCP sont le signe le plus tangible de leur productivité, elles n'apparaissent pour la plupart que tardivement, alors que le programme est déjà clos. C'est le cas notamment du numéro de L'Espace géographique sur «le paysage et son concept » en 1973, pour la RCP 231 et le CIMA, et des publications aux éditions du CNRS déjà citées, Espaces périphériques (1978) et Espaces vécus et civilisations (1982). Cependant une littérature grise, éventuellement des articles de revue, ont pu accompagner l'avancée des recherches. Il en est ainsi, par exemple, de la série des Remica, à partir de 1973, et de l'article "Systèmes spatiaux et structures régionales " ${ }^{12}$, pour la RCP Kayser ${ }^{13}$; de la participation des membres de la RCP Frémont au débat «Paysages et sémiologie » dans L'Espace géographique en $19744^{14}$. 


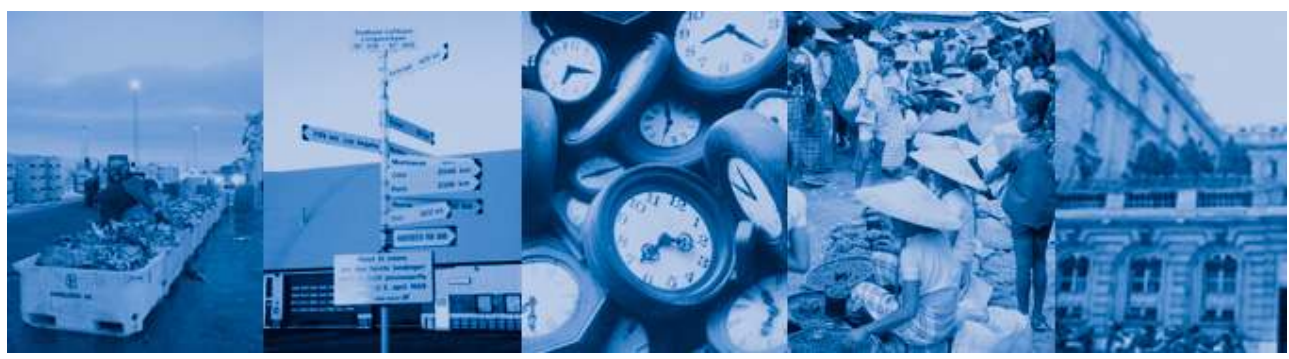

(c) CNRS Photothèque

Mais une dimension importante du travail effectué échapperait largement si l'on se limitait à ses formes les plus diffusées. Dans une période marquée par des développements techniques (émergence de l'informatique) et méthodologiques nouveaux, et par un effort de systématisation des enquêtes, un objectif central fut d'établir des inventaires raisonnés. "Dépouillement systématique de la littérature française et étrangère touchant à l'organisation de l'espace" et conversion en fiches mécanographiques pour la RCP Brunet, ainsi qu'une enquête sur les banques de données, diligentée par Sylvie Rimbert, chercheuse à l'ERA 214 de Strasbourg; inventaire des établissements industriels du Midi français et de la Catalogne pour la RCP Kayser, avant une recherche lexicale sur les syntagmes comprenant "espace " dans les sciences sociales; typologisation des «distances » et des «espaces" pour la RCP Frémont; recherches diverses sur le vocabulaire de la géographie pour la RCP Pinchemel. Cette multiplication des inventaires est tout à fait caractéristique de la période en géographie et on la retrouverait ailleurs. Elle manifeste le souhait d'élaborer des bases de travail collectives. Elle accompagne une dimension commune: des préoccupations réflexives, voire épistémologiques - ou du moins lexico-sémantiques ${ }^{15}$. Celles-ci revêtent cependant des niveaux d'intensité et des modalités fort variables. Le souci épistémologique peut être directement inscrit dans un souci de refondation de la discipline («Les systèmes d'organisation de l'espace ») ou d'analyse de celle-ci (RCP Pinchemel). Il vise davantage l'élaboration d'un concept nouveau (l'» espace vécu ») dans la RCP Frémont, à partir de travaux empiriques à priori éloignés les uns des autres. Dans le cas de la RCP Kayser, ce sont les processus politiques et économiques de production de l'espace - dans des "espaces périphériques " aussi bien urbains que ruraux -, dont on recherche l'explicitation; ici, le caractère collectif du travail de terrain est une autre dimension majeure. Par-delà leurs convergences, ces RCP manifestent bien la diversité des postures et le foisonnement des centres d'intérêt qui traversent la géographie française à partir de ces années-là ${ }^{16}$.

Au-delà des RCP...

Les RCP ont constitué un moment initial de recherche collective qui comporte des prolongements dans les années suivantes. En effet, la plupart de leurs participants se retrouvent, parfois avec d'autres, dans les GRECO (Groupe de recherches coordonnées) et ATP (Action thématique programmée) du CNRS ; par exemple, le GRECO « Histoire du vocabulaire scientifique» (1980-1982) pour la RCP Pinchemel, le GRECO «Processus d'urbanisation» (1977-82) pour la RCP Kayser, l'ATP «Observation continue du changement social et culturel » pour beaucoup d'entre eux. Et une partie de ces équipes accède rapidement au statut d'ERA du CNRS. On peut considérer finalement que les RCP ont accompagné, et dans une certaine mesure, contribué à une structuration plus générale de la recherche en géographie, dans une période où la discipline connaissait des transformations décisives. 


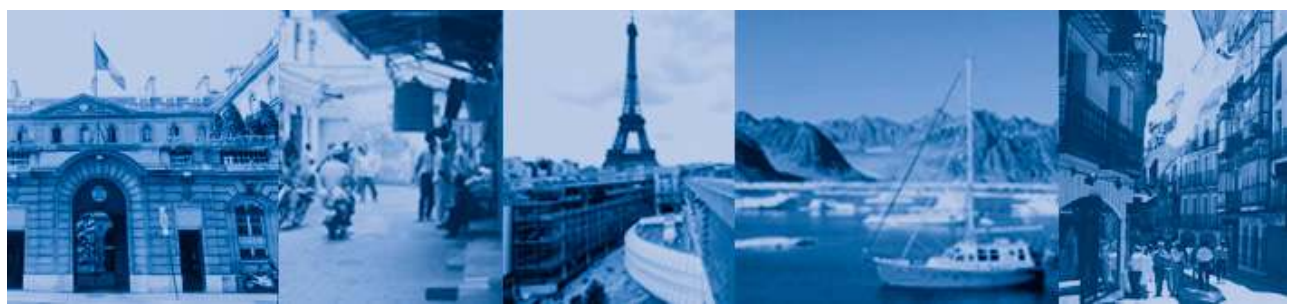

(C) CNRS Photothèque

\section{NOTES}

1.Nous entendons par là celles qui se sont institutionnalisées avant la fin du XIX ${ }^{\mathrm{e}}$ siècle et de manière durable.

2.Ce qui, étant donné les effectifs, implique en province la participation de professeurs de différentes universités.

3.Il en existait trois à la fin des années 1960 : le Service de documentation et de cartographie géographique (Paris, initialement dirigé par Jean Dresch), le laboratoire de géomorphologie de Caen (fondé par André Journaux) et le CEGET de Bordeaux (Guy Lasserre).

4.Sous réserve d'inventaire supplémentaire.

5.Dans les lignes et les notes qui suivent, nous mentionnons un certain nombre de participants à ces quatre RCP. Mais il ne s'agit en aucune façon de listes à prétention exhaustive : établies à partir du croisement des informations de sources hétérogènes archives, entretiens, publications... qui ne coïncident pas toujours, en raison notamment des décalages chronologiques -, elles omettent inévitablement quelquesuns de celles et ceux qui ont pris une part active à ces recherches collectives. 6.Participent notamment à cette RCP : É. Juillard (Strasbourg), patron de l'ERA 214, et des membres de son équipe ; O. Dollfus (Paris VII), Gilles Sautter (Paris I), A. Fel (Clermont-Ferrand), P. Claval (Besançon), J.-L. Piveteau (Fribourg), J.-B. Racine (Montréal). L'année suivante (1971) s'y associera le Centre d'analyse de l'espace (Paris I) dirigé par J. Beaujeu-Garnier. C'est surtout un club de jeunes professeurs novateurs, même si quelques assistants de Reims (A. Reynaud) ou Strasbourg (É. Dalmasso) y ont participé. La RCP 256 n'a pas été renouvelée du fait des tâches nouvelles qui ont occupé R. Brunet peu après : création et animation de la revue L'Espace géographique (à partir de 1971-1972), départ de Reims pour Paris et le CNRS (après 1974-1975).

7.Figurent parmi les participants : J.-Ph. Beau, J.-P. Ferrier, N. Girard, R. Perrin, J. Richez (Aix et Marseille), P. Barrère, M. Cassou-Mounat (Bordeaux), A. Boudou, M. Cohou, G. Jalabert, J. Pilleboue (Toulouse), H. Capel, E. Giral, E. LLuch (Barcelone). 8.Les autres participants à la RCP et signataires de l'ouvrage collectif sont : J. Chevalier (Caen, puis Le Mans) et A. Metton (Orléans).

9. Outre des géographes tels V. Berdoulay (alors à Berkeley), P. Claval et F. Carré (Paris IV), N. Broc (Perpignan), J.-P. Nardy (Besançon), G. Pinchemel (Paris I), A. Reynaud (Reims), M.-C. Robic (Paris XII), participent à cette RCP une historienne, G. Aujac 
(Rennes), et des représentants du Département des cartes et plans de la Bibliothèque nationale. S'y adjoindront ensuite J.-L. Tissier, A.-M. Briend, M.-V. Ozouf-Marignier, G. Nicolas, D. Loi, G. Palsky...

10.Géographe, directeur adjoint du CNRS pour les sciences humaines entre 1963 et 1972.

11.Lévy J., « Roger Brunet », in Julliard J. et Winock M., Dictionnaire des intellectuels français, Le Seuil, 1996, pp. 192-193.

12.REMICA, « Systèmes spatiaux et structures régionales 》, Espaces et sociétés, 1974, $\mathrm{n}^{\circ} 12$.

13.18 numéros jusqu'en 1983, date à laquelle la RCP est évidemment achevée en tant que telle.

14. $\mathrm{N}^{\circ} 2$ : articles d'A. Frémont, A. Metton et M.-J. Bertrand.

15.Cf. Orain 0 ., « La géographie comme science. Quand 'faire école' cède le pas au pluralisme » in Robic M.-C., dir., Couvrir le monde. Un grand XXe siècle de géographie française, Paris, ministère des Affaires étrangères, Association pour la diffusion de la pensée française (ADPF), 2006, spécialement pp.111-114.

16.Notons que ceux-ci peuvent se retrouver en partie au sein même des groupes constituant les RCP ; car ce qui fédère peut relever du thématique, sans nécessairement impliquer l'accord sur une orientation problématique. D'où l'intérêt de la discussion collective...

\section{RÉSUMÉS}

Jusqu'à la fin des années 1960, le CNRS représentait principalement pour les géographes français un lieu d'hébergement dans le cadre duquel ils pouvaient achever une thèse d'État. Mais à la fin de la décennie, une vision nouvelle du travail d'équipe, rompant avec l'individualisme universitaire jusque-là de mise, s'est partiellement imposée. Olivier Orain et Marie-Pierre Sol font un retour historique sur cette période.

Until the end of the 1960s, French geographers were used to consider CNRS mainly as a place where to achieve their PhD. Later on, a new vision of teamwork appeared, quitting academic individualism. A flash back by Olivier Orain and Marie-Pierre Sol.

\section{INDEX}

Mots-clés : ATP, géographie, Greco, recherche coopérative sur programme, RCP, groupe de recherches coordonnées, action thématique programmée 


\section{AUTEURS}

\section{OLIVIER ORAIN}

Olivier Orain est chargé de recherche au CNRS, membre de l'UMR 8504, « Géographiecités », équipe « Épistémologie et histoire de la géographie » (E.H.GO).

MARIE-PIERRE SOL

Marie-Pierre Sol est professeur agrégé à l'université de Toulouse-Le Mirail et associée à l'UMR 8504. 